fluid, and the vessels of the plexus were remarkably congested. No signs of bone disease or fracture were observed, nor was there any ear disease or tubercle.

Newcastle-on-Tyne.

\section{SOME POINTS IN THE TREATMENT OF ACNE, ESPECIALLY OF THE FACE.}

BY JOHN KENT SPENDER, M.D.LOND., PHYSTOLAN T'O THE MINERAL WATER HOSPITAL, BATE.

IF a doctor may strive to rival an artist in idealising the human face, there are few functions for which he will earn stronger gratitude than in removing the traces of disfigurement and deformity. There are, indeed, deformities which have almost classical tradition to consecrate them; and there is a native ugliness which may be to some extent glorified by the powerful lineaments of emotion and character; but most disfigurements are associated with pettiness or vulgarity, and there are few consolations for the unhappy man or woman who has a big red nose or a heavy crop of pimples on the forehead. Pimples, though veiled under the elegant Greek of the word "leichen" $(\lambda \epsilon \hat{\imath} \chi \eta \nu)$, are supposed to denote the glutton or the winebibber, and to be beyond the rhetoric of the most subtle special pleader: for instance, if they are red, they are said to tell the tale of excess; if dotted with black spots, they proclaim uncleanliness and struma; and if only pale and insignificant, there is always the dark hint of a bad constitution in the background. Popular pathology is not always logical; and it would be difficult to convince some patients that their skin trouble is, after all, a purely local affair, depending upon inflammation or inaction of the small cutaneous glands. The purifying action of the skin is an obscure dogma to the popular understanding; and therefore people are slow to take in the fact that when Nature is baffled in her duties by too much wind, or dust, or sun, and too little soap and water, she takes her revenge by desquamations and efflorescences, and perhaps more serious things than these.

Acne (the Greek original means the "bloom of anything") is the term given to a retention of the secretion of the sebaceous glands of the skin, with secondary inflammation and deposit in them and in the hair follicles. It is characterised by red conical or hemispherical elevations or nodules; some are solid, others are filled with pus; they are found everywhere except upon the palms and soles, and affect chiefly the skin of the face, chest, and back; and they occur mostly in young persons. The disease appears to be due to the occlusion of the orifice of the hair follicles or of the ducts of the sebaceous glands opening into them. The retained secretion then becomes a source of irritation and inflammation, and suppuration follows in and around the hair sac and its appendages. ${ }^{1}$ This, expressed in the fewest words, is the pathological anatomy of simple or uncomplicated acne.

Omitting for the sake of brevity and clearness all the intermediate forms, we may go at once to a type of disease at the other end of the scale, and speak of the so-called acne rosacea. This attacks the face and scalp alone, and is characterised by an intense reddening of the skin, due to an injection of the bloodvessels, without much swelling or tension. The serpentine vascular lines, the blood in which may be momentarily driven out by pressure, are most abundant on the sides and the bridge of the nose. This is an obstinate disease, occurring chiefly in advanced age, though not unknown in youth. However great may be the hypertrophy of the skin the disease never extends deeper than the skin, nor does it lead to ulceration. ${ }^{2}$ Between the extreme phases of local disease thus delineated there are countless grades and shades which ought to be recognised; for they are so many tokens of constitutional power or inertness, as the case may be.

My notes of treatment profess, then, to deal only with acne punctata, acne vulgaris or indurata, and the acne rosacea, which betrays a grave alteration in the nutritive function of the skin.

The object of the practitioner should be to prevent acne

1 New Sydenham Society's Lexicon of Medicine and the Allied Sciences. punctata from passing on to acne vulgaris by getting rid of: obstruction in the glands and checking the hyperæmic condition; in acne indurata, to lessen hyperæmia and promote the absorption of inflammatory products; and in acne rosacea to destroy the new growth of connective tissue.

The therapeutic points may be expressed thus:-Quiet dormant acne requires stimulation and a spur to more healthy action, while those species of acne which are marked by heat and tension call for soothing local measures. In the one case the medicinal and dietetic plan should be tonic and supporting; in the other we should advise a combination of tonic and aperient remedies and a cooling abstemious regimen.

In order to promote a healthy action of the sebaceous. glands, and to prevent the formation of what are called "comedones," the following plan should be adopted, as originally described by $\mathbf{D r}$. Liveing. The steps of his method are as follows: (a) Steam the face every night by holding it over a basin of hot water for a few minutes. $(b)$ Rub the skin for five or ten minutes with soap (I prefer terebene soap) and flannel, or with a soft nail-brush ; then sponge off the soap with warm water. (c) When the face has been dried, a lotion should be thoroughly applied, composed as follows: half an ounce of precipitated sulphur, two drachms of glycerine, one ounce of spirits of wine, with three ounces each of rose-water and lime-water. This is allowed to dry on the skin and to remain on all night. In the morning the face is cleansed with warm oatmeal and water or weak gruel. If, for any reason, an ointment seems preferable to a lotion, a combination of precipitated sulphur and vaseline is very useful. The treatment must be modified or suspended for two or three nights if the skin becomes sensitive and somewhat tender. Dr. Liveing contends that the most common cause of failure is want of perseverance or timidity on the part of the patient or of the doctor; and that we ought not to be frightened from continuing efficacious remedies by a temporary increase in the redness and irritatability of the skin. ${ }^{3}$ If, by any chance, this plan be unsuccessful, nothing is so effective as the application of potash soap in the form of a lotion. The lotion is composed of one ounce of soft soap, one ounce of rectified spirits of wine, and seven ounces of rose (or distilled) water. This should be rubbed in vigorously with a piece of flannel for a short time, taking care not to make the skin sore. According to Dr. Liveing, the worst cases of acne will yield to the soft soap treatment if practised with necessary caution.

For eight years and more $I$ have followed in its main outlines Dr. Liveing's plan, and with great success. But the experience which only actual work brings has led me to make modifications to suit the various susceptibilities and irritabilities of human nerves and skin. Some of these modifications I will now relate in the fewest words. In the first place, something is often required to be done during the daytime to pacify the heat and throbbing, which are part of the usual history of acne. An excellent lotion, the heritage of nearly every dermatologist, is made by combining oxide of zinc, calamine, prepared chalk, lead lotion and lime water, to which may be added a small quantity of glycerine. Let the bottle containing this be gently waved about so as to diffuse the materials, which are only held in suspension; then pour a little into a saucer, and with a sponge (reserved for the purpose) sprinkle the face from time to time. Wipe off, when necessary, with a bit of fine muslin the powder which remains on the skin after the evaporation of the fluid, and the face may be washed occasionally with a little starch gruel. In the second place the cases are not a few in which it is better not to use any kind of soap as part of the evening ceremonial. After the face has been steamed, we may put on a medicated jelly composed of oxide of zinc, gelatine, and glycerine. It must be liquefied by putting the vessel that holds it into hot water, and then applied with a brush. Then, thirdly, there are some sensitive skins intolerant of sulphur in any guise. When this is so, we should think of combinations of lead and chalk and zinc, blended as a quasi-ointment with the finest vaseline. There are several pharmaceutists in London and the provinces who prepare oleate of lead and oleate of bismuth; and Dr. McCall Anderson's formula of oleate of bismuth with vaseline and white wax has been aptly described as "one of the most healing of salves." Sometimes nothing agrees better than the old-fashioned but 
capital substance called Kirkland's " neutral cerate," which is composed essentially of lead plaster and olive oil. Whatever combination may be chosen (and only a ripe experience with a sound judgment can decide), the ointment should be smeared over all the affected parts of the face immediately after the process of steaming, and allowed to remain during the whole night. For the acne which is often a trouble to young women at the time of commencing menstruation I prescribe the following ointment with much confidence:Ammoniated mercury, a scruple; precipitated sulphur, a drachm; oil of the sweet almond, half an ounce; and white vaseline to make two ounces. By methods such as the above, industriously carried out, an ugly, blotchy face may be cormonly restored to a fair share of comeliness.

In the treatment of acne there is still room for those emollient "dusting powders" which once occupied an important place in the therapeutics of the skin. Equal quantities of oleate of zinc and pulverised starch, with a small percentage of precipitated sulphur, may be put on several times a day after moistening the surface with a warm damp sponge. Now and then a quiet acne may disappear entirely in a few weeks without any other application. But the extreme forms of the so-called acne rosacea require a whole battery of new remedial forces, as it seldom yields to mild and coaxing means. I refer with much satisfaction to a paper published in the early part of 1885 by Mr. Tom Robinson.* $\mathrm{He}$ advises no parley with an enemy which cannot be tamed or dislodged after inflammatory hyperplasia of connective tissue has once been set up. When inflamed papules are developed, a lotion should be applied occasionally, composed of bisulphuret of mercury and almond emulsion (two grains of the former to one ounce of the latter). When the congestive process goes on to suppuration the face may be fomented with hot water; and after the acute stage has subsided a combination of a scruple of yellow oxide of mercury and one ounce of lard ought to be rubbed into the face, and continued every night so long as it may seem necessary. $\mathrm{Mr}$. Robinson's favourite means for tranquillising an angry skin is a lotion composed of bismuth and glycerine of starch, Aessening the starch by three-fourths.

It is time to say a few words about internal remedies. It is a pity that so much attention must be given to the diet and the details of cooking, as this is the real impediment in many instances to the attainment of even a moderate success. All food which is difficult to digest must be avoided, as the waste stuff which it contains is a source of physiological irritation and worry. Simplicity will best suit that "conscience of the body" called the stomach; and regularity in the meals is of high importance. Alcohol and tobacco are usually superfluous, and sometimes poisonous; but concerning the former an exception may be made in the wase of strumous young men and women, to whom a little beer (taken with meals) is often quite valuable. I must insist upon the daily constitutional walk as a necessary article of faith and practice; and I venture to add that systematic writers on diseases of the skin do not lay sufficient stress on the proper ventilation of houses and rooms (especially the bedroom) as essential to the respiratory function of the skin. This function is thwarted and blocked when the surrounding air is deficient in quantity and chemically bad in its quality; and we cannot wonder at the prevalence of acne and other similar skin diseases, when we call to mind the polluted elements which some people love to breathe.

With regard to the Pharmacopoia, the rule is almost absolute that arsenic is nearly the last drug which a practitioner should think of. Combined with perchloride of mercury, arsenic has a useful place in the management of dull inert acne in plethoric persons. Iron is often necessary, and can be conveniently given in one of two forms: either (a) as the ammonio-citrate, with an effervescing draught of citric acid and bicarbonate of potash; or $(b)$ as the sulphate of iron, with dilute sulphuric acid and sulphate of magnesia. The mild aperient action of the latter combination is a distinct help; and further aid in this direction is afforded by the pill of aloes and myrrh. Residents in Bath can enjoy a regular and prolonged administration of the thermal waters. It is lucky that there are no reputed "specifics" in the treatment of acne; there is less scope for secret nostrums and remedies of doubtful fame; and the culture of the "general health" becomes not a vague and occult symbol, but the expression of an axiomatic truth."

F'alstaff calls Bardolph's red nose "a perpetual triumph, an everlasting bonfire-light." Most people are only too glad to get rid of this uncanny illumination. The cure or alleviation of an old acne on the face is sometimes such a transfiguration from ugliness to comparative beauty as to bring credit to the medical adviser, gratitude from the patient, and a substantial addition to the historic glory of therapeutic art.

\section{THE TREATMENT OF INTUSSUSCEPTION.}

\section{BY F. H. RLLIOTT, M.D.}

THE condition known as intussusception is at all times so serious, and in so many cases fatal, that it is incumbent upon those who have had experiences similar to those recorded in THE LANCET of Oct. 23rd by Dr. Cheadle to place them on record. The following case occurred in private practice, and the facts are given from rough notes taken at the time.

On Sunday, Nov. 8th, 1885, I was sent for to see the child (eight months old) of a friend. The child, it appeared, had been quite well until the previous day, whon it had been sick, tor which a dose of castor oil had been given, after which a little blood had been passed by way of the bowels. When I saw it on the Sunday morning there was sickness with frequent straining, and the passage of small quantities of blood and mucus. The case appeared at first to be one of dysenteric diarrhœa, produced probably by improper feeding Sc.; but on a second visit, however, the same day, I made a more careful examination, and, in addition to a sausage-like tumour, which could be distinctly felt in the left iliac region, I discovered the presence of the intussusception about three inches up the rectum. Most fortunately, I was able to avail myself of the advice and assistance of my friend Dr. G. V. Poore, who on arrival confirmed the diagnosis. By this time, in addition to the abore symptoms, the child was much exhausted, and evidently in a critical condition. We decided to see what could be done for its relief, and in this we received great assistance from the father, who behaved throughout with much self-restraint and judgment. Chloroform being administered, the father held the child up by the legs, so that the force of gravity might be turned to account, and I gently inflated the bowel by means of an ordinary pair of bellows, whilst Dr. Poore kneaded the abdomen. After the lapse of a few minutes intestinal morement was audible, and on examination neither the sausage-like swelling in the iliac region nor the tumour in the rectum could be discovered, and in a short time after recovery from the chloroform it was evident that the general state of the child had considerably improved. By the next morning, however, all the signs and symptoms had returned, and the child was as bad as-indeed, worse than-before. Regarding it as a hopeless case, I did not propose to proceed further; but as a day or so passed without death resulting, I determined to repeat the operation. The inflation with the bellows this time had no effect. The tumour in the rectum did not move. I therefore fixed an indiarubber tubing on to an ordinary funnel, and, the child being inverted and the end of the tubing inserted in the bowel, had warm water poured from a considerable height, as recommended by Dr. Day in his work on "Diseases of Children." This had the effect of displacing the intussusception, but not really overcoming it, as a swelling could still be detected an inch or so abore the left iliac region; I therefore fixed the indiarubber tubing on to the bellows, and passed it, well oiled, gently up the bowel until it met the obstruction. I then inflated, and in the course of a minute or so a sudden and general distension of the whole abdomen told me that the passage had been restored. The vomiting, tenesmus, \&c., were almost at once relieved; milk with lime-water was retained by the stomach, and quiet, refreshing sleep ensued. For five days, although no motion was passed, everything went well; but on the evening of the fifth day the child suddenly began to shriek as if in great pain, and the whole of the symptoms and physical signs of intussusception returned. I at once took the same

5 I am imaging an ideal patient who has no special diathetic tendency, as any complication of gout or of syphilis must be treated on its
own merits and needs. 